\title{
Dynamic hyperinflation impairs daily life activity in asthma
}

\author{
Akke-Nynke van der Meer ${ }^{1}$, Kim de Jong (i) ${ }^{1}$, Aranka Hoekstra-Kuik ${ }^{1}$, \\ Elisabeth $\mathrm{H}$. Bel ${ }^{2}$ and Anneke ten Brinke ${ }^{1}$
}

Affiliations: ${ }^{1}$ Medical Centre Leeuwarden, Leeuwarden, The Netherlands. ${ }^{2}$ Academic Medical Centre, Amsterdam, The Netherlands.

Correspondence: Akke-Nynke van der Meer, Henri Dunantweg 2, 8934 AD Leeuwarden, The Netherlands. E-mail: a.n.van.der.meeraznb.nl

\section{@ERSpublications}

Dynamic hyperinflation is associated with impaired daily life activity in asthma and is therefore an important target for treatment http://ow.ly/yBIR30nlKx0

Cite this article as: van der Meer A-N, de Jong K, Hoekstra-Kuik A, et al. Dynamic hyperinflation impairs daily life activity in asthma. Eur Respir J 2019; 53: 1801500 [https://doi.org/10.1183/13993003.01500-2018].

\section{ABSTRACT}

Introduction: Dynamic hyperinflation has been documented in asthma, yet its impact on overall health and daily life activities is unclear. We assessed the prevalence of dynamic hyperinflation in moderate to severe asthma and its relationship with the scores of a set of specific and general respiratory health questionnaires.

Methods: 77 nonsmoking asthma patients (Global Initiative for Asthma steps 4-5) were recruited consecutively and completed five questionnaires: Asthma Control Questionnaire, Clinical COPD (chronic obstructive pulmonary disease) Questionnaire, St George's Respiratory Questionnaire, London Chest Activity of Daily Living scale (LCADL) and Shortness of Breath with Daily Activities (SOBDA). Dynamic hyperinflation was defined as $\geqslant 10 \%$ reduction in inspiratory capacity induced by standardised metronome-paced tachypnoea. Associations between level of dynamic hyperinflation and questionnaire scores were assessed and adjusted for asthma severity.

Results: $81 \%$ (95\% CI 71.7-89.4\%) of patients showed dynamic hyperinflation. Higher levels of dynamic hyperinflation were related to poorer scores on all questionnaires $(r=0.228-0.385, p<0.05)$. After adjustment for asthma severity, dynamic hyperinflation remained associated with poorer scores on LCADL $(\mathrm{p}=0.027)$ and SOBDA $(\mathrm{p}=0.031)$.

Conclusion: Dynamic hyperinflation is associated with poorer overall health and impaired daily life activities, independent of asthma severity. Because of its major impact on everyday life activities, dynamic hyperinflation is an important target for treatment in asthma. 


\section{Introduction}

Asthma is a heterogeneous condition of the airways with many clinical and inflammatory sub-phenotypes [1]. In day-to-day practice these different subtypes may present with different asthma symptoms. A subset of asthma patients report exercise intolerance and limitations in daily life activities as the most prominent symptoms, rather than the classical wheezy attacks, with a major impact on their quality of life [2]. Many factors may contribute to these exercise and activity limitations in asthma, including psychological factors, respiratory muscle strength, bronchoconstriction and dynamic hyperinflation [3].

Dynamic hyperinflation, described as an increase in end-expiratory lung volume under conditions of greater minute ventilation (e.g. exercise), is a well-known feature in chronic obstructive pulmonary disease (COPD) resulting from reduced expiratory airflow [4]. It is strongly related to exertional dyspnoea and diminished daily physical activity in COPD patients $[5,6]$. Interestingly, dynamic hyperinflation is not exclusive to COPD, and a few small studies suggest it to be present in asthma as well. Dynamic hyperinflation has been reported in patients with stable asthma following methacholine provocation $[7,8]$ or exercise testing [9], probably reflecting induced bronchoconstriction. Recently, it was suggested that dynamic hyperinflation is particularly important in patients with more severe asthma. These patients have been shown to develop dynamic hyperinflation during exercise to the same magnitude as COPD patients with similar degrees of airway obstruction [10]. Unlike COPD, it has been suggested that in asthma ongoing inflammation may impair small airway function [11]. In patients with late-onset asthma, air trapping was markedly present, and associated with systemic eosinophilic inflammation and severe exacerbations [12]. Moreover, in a small unblinded study in 10 patients with moderate to severe allergic asthma, the extent of dynamic hyperinflation decreased with omalizumab treatment, which corresponded to an improvement in symptoms and exercise capacity [13]. So, ongoing small airway inflammation might promote the development of dynamic hyperinflation and play a role in daily exercise limitations in subsets of asthma patients. Importantly, dynamic hyperinflation is found to be greater in obese versus nonobese asthma patients, whether or not related to reduced chest wall compliance, and therefore obesity has to be taken into account when evaluating dynamic hyperinflation in asthma [14]. Whatever the underlying mechanism, so far little is known about the prevalence of dynamic hyperinflation in moderate to severe asthma and in particular its impact on asthma symptoms, activities of daily life and perceived wellbeing.

Although asthma symptoms, limitations in daily life activities and reduced quality of life are all important outcomes from the patient's perspective, not all are taken into account equally well in asthma management. Asthma symptoms are generally scored using the Asthma Control Questionnaire (ACQ) or Asthma Control Test, focusing on classical symptoms of variable dyspnoea and wheezing and with only little emphasis on symptoms of exercise intolerance and limitations in daily life activity. The latter symptoms might better be detected by questionnaires used for COPD patients. This is supported by observations in recent studies on the effects of mepolizumab in severe eosinophilic asthma patients, showing greater improvements in the St George's Respiratory Questionnaire (SGRQ) score than in the ACQ score $[15,16]$.

These observations stress the need to investigate the role of dynamic hyperinflation in asthma symptoms and limitations in daily activities, in particular in patients with more severe disease. If dynamic hyperinflation indeed explains important patient-related outcomes this could become an important target for treatment of asthma. Therefore, in the present study we primarily investigated the prevalence of dynamic hyperinflation in moderate to severe asthma. As a secondary objective, we assessed the relationship between the degree of dynamic hyperinflation and severity of respiratory symptoms and limitations of daily life activities derived from different specific and general respiratory health questionnaires.

\section{Methods}

\section{Patients}

Patients with moderate to severe asthma (age $\geqslant 18$ years) were recruited from a nonacademic pulmonary outpatient department in the Netherlands (Medical Centre Leeuwarden) between June 2016 and January 2018. 77 patients were consecutively included in the present study, which is part of an extensive research programme aimed at exploring the clinical relevance of dynamic hyperinflation in asthma. All patients were on regular treatment with medium to high doses of inhaled corticosteroids (ICS) $\left(\geqslant 500 \mu \mathrm{g} \cdot \mathrm{day}^{-1}\right.$ fluticasone or equivalent) with or without daily oral corticosteroids, combined with long-acting $\beta$-agonists or other controller for $\geqslant 6$ months, according to the Global Initiative for Asthma steps $4-5$ [17]. All patients had stable asthma without exacerbations during the 4 weeks before inclusion, were nonsmokers (smoking history $\leqslant 10$ pack-years), had a body mass index $(\mathrm{BMI}) \leqslant 30 \mathrm{~kg} \cdot \mathrm{m}^{-2}$, and airway obstruction with a forced expiratory volume in $1 \mathrm{~s}$ (FEV1)/forced vital capacity $\leqslant 80 \%$ predicted. Patients with concurrent respiratory disease, major unrelated comorbidities and pregnancy were excluded. The study was approved by the local medical ethics committee and all patients gave their written informed consent. The trial is registered at the Netherlands Trial Register (identification number NTR5873). 


\section{Methods and design}

For the present study all measurements were performed during one visit. First, data on all relevant patient characteristics, medication use and asthma related healthcare use in the previous year were collected by the investigator. Then, the patients completed a set of five specific and general respiratory health questionnaires. Finally, they performed lung function tests and had blood drawn for cell differential counts and total immunoglobulin E levels.

\section{Questionnaires}

All subjects completed a set of five respiratory health questionnaires: ACQ-6 (range 0-6), focusing on asthma control [18]; the Clinical COPD Questionnaire (CCQ) (range 0-6), focusing on COPD control [19]; SGRQ (range 0-100) [20], focusing on respiratory symptoms, quality of life and limitations in daily life activities; and the London Chest Activity of Daily Living (LCADL) questionnaire (range 0-75) [21], and the Shortness of Breath with Daily Activities (SOBDA) questionnaire (range 1-4) [22], both focusing on limitations in daily life activities. The ACQ is validated in asthma patients; the other questionnaires used in this study are validated in COPD patients.

\section{Lung function}

Pulmonary function tests included exhaled nitric oxide fraction measurement [23], spirometry and body plethysmography [24]. Bronchodilators were withheld before pulmonary function tests for $\geqslant 6$ and $\geqslant 12 \mathrm{~h}$ for the short-acting and long-acting $\beta_{2}$-agonists, respectively.

\section{Dynamic hyperinflation}

To test for dynamic hyperinflation, all subjects underwent metronome-paced tachypnoea measurement (MPT) [25]. Subjects were seated, breathing through a mouthpiece connected to the spirometer (MasterScreen-PFT; Jaeger, Mettawa, IL, USA) and were instructed on the performance of the inspiratory capacity manoeuvres. At the start of this test the baseline inspiratory capacity was measured as the mean of three acceptable inspiratory capacity manoeuvres while the patient was at rest. Subjects were then asked to breathe at a metronome-paced frequency of twice the resting breathing rate for $20 \mathrm{~s}$, and immediately afterwards an inspiratory capacity manoeuvre was performed [26]. The procedure was repeated after subjects had returned to their resting breathing level. Subjects were encouraged to maintain a stable tidal volume. Dynamic hyperinflation was calculated as the difference between the inspiratory capacity measured during increased pacing and the inspiratory capacity at rest. A decrease of $\geqslant 10 \%$ in the inspiratory capacity was considered as dynamic hyperinflation [26].

\section{Statistical analyses}

Differences between subjects with and without dynamic hyperinflation were analysed using unpaired t-tests, Mann-Whitney U-tests or Chi-squared tests, wherever appropriate. Subsequently, linear associations between the MPT-induced degree of dynamic hyperinflation and the questionnaire scores were assessed using Spearman rank correlations. Finally, we assessed whether univariate associations remained when adjusting for asthma severity parameters (ACQ score, fluticasone equivalent dose and FEV1 \% pred) using multivariable linear regression models. All analyses were performed using SPSS software (version 20; IBM, Armonk, NY, USA). Two-sided p-values $<0.05$ were considered to be statistically significant.

\section{Results}

Patient characteristics and dynamic hyperinflation

77 patients with moderate to severe asthma participated in the study. Patient characteristics are shown in table 1 . The majority of patients was male and had adult-onset asthma. Only $31 \%$ of patients had smoked previously. Despite the limited cigarette exposure in the past (median (range) 0 (0-10) pack-years), the degree of airway obstruction varied with $\mathrm{FEV}_{1}$ values ranging from $26 \%$ pred to $104 \%$ pred.

In the whole group of 77 patients, the median (range) of MPT-induced reduction in inspiratory capacity was $0.47(-0.42-1.57) \mathrm{L}$ or $17.8(-14.1-47.2) \%$ from baseline. According to the predefined cut-off level, 62 out of 77 patients $(80.5 \%, 95 \%$ CI $71.7-89.4 \%)$ showed dynamic hyperinflation, with a median (range) MPT-induced reduction in inspiratory capacity of $0.55(0.19-1.57)$ L or $19.5(10.2-47.2) \%$ change from baseline inspiratory capacity. The other 15 patients showed no dynamic hyperinflation with a median (range) reduction in inspiratory capacity of only $0.16(-0.42-0.35) \mathrm{L}(4.8(-14.1-9.0) \%)$. When comparing the two groups, patients with dynamic hyperinflation reported significantly more visits to the pulmonologist in the preceding year $(\mathrm{p}=0.031)$ and tended to use higher daily doses of inhaled and oral corticosteroids. There was no difference between the groups in smoking history or atopic status, nor was there a difference in blood eosinophil counts. At baseline, patients with dynamic hyperinflation had lower levels of FEV1 and showed more severe air trapping and static hyperinflation. 
TABLE 1 Characteristics of moderate to severe asthma patients with and without dynamic hyperinflation

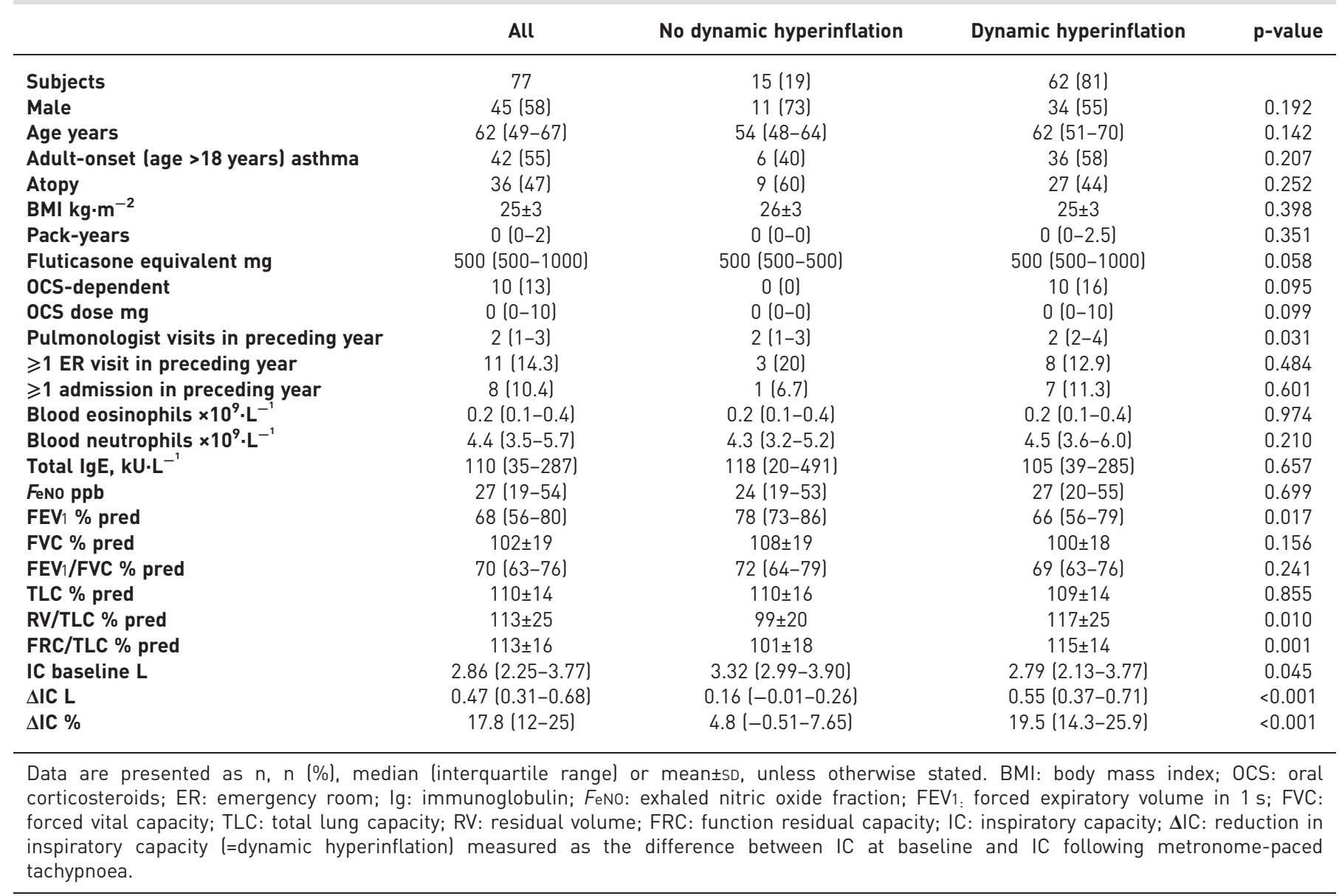

Dynamic hyperinflation and symptom scores

All patients completed the five questionnaires. The scores in ACQ and CCQ were not significantly different between patients with and without dynamic hyperinflation (table 2). In the group with dynamic hyperinflation $42 \%$ of the patients had an ACQ score of $\geqslant 1.5$ versus $27 \%$ of the patients in the group without dynamic hyperinflation $(\mathrm{p}=0.179)$. The patients with dynamic hyperinflation showed a poorer score on the LCADL $(\mathrm{p}=0.0031)$ and a trend towards a poorer score on the SGRQ $(\mathrm{p}=0.070)$ and SOBDA $(\mathrm{p}=0.094)$.

When analysed linearly, the scores of all the questionnaires were significantly related to the MPT-induced degree of dynamic hyperinflation, as shown in figure 1. Higher levels of dynamic hyperinflation corresponded with poorer scores on all five questionnaires. Information on the association between dynamic hyperinflation and the subdomains of the questionnaires is presented in supplementary table S1. For $\mathrm{FEV}_{1} \%$ pred, there was no significant correlation with any of the questionnaire scores $(\mathrm{p} \geqslant 0.13)$. Nor were parameters of static hyperinflation associated with questionnaire scores, except for the SOBDA questionnaire (supplementary table S2).

After adjustment for parameters of asthma severity (ACQ score, FEV1 \% pred, ICS dose), dynamic hyperinflation remained associated with poorer scores on LCADL $(\beta=0.198, p=0.044)$ and SOBDA $(\beta=0.016, p=0.009)$, but not with the other questionnaires (table 3 ).

\section{Discussion}

In the present study, we showed that dynamic hyperinflation is a relevant feature in moderate to severe asthma. The proportion of patients with dynamic hyperinflation was high. The severity of dynamic hyperinflation was related to lower scores on five different respiratory health questionnaires and significantly associated with impaired daily life activities. These results suggest that dynamic hyperinflation may be an important target for treatment in moderate to severe asthma. 
TABLE 2 Respiratory symptom scores in asthma patients with and without dynamic hyperinflation

\begin{tabular}{lccc} 
& No dynamic hyperinflation & Dynamic hyperinflation & p-value \\
\hline Subjects & $15(19)$ & $62(81)$ & \\
ACQ $^{\#}$ & $0.8(0.7-1.7)$ & $1.3(0.8-2.0)$ & 0.144 \\
CCQ $^{\#}$ & $1.1(0.6-1.8)$ & $1.2(0.7-2.1)$ & 0.234 \\
SGRQ $^{\#}$ & $25.3(13.6-30.1)$ & $28.8(15.9-45.3)$ & 0.070 \\
LCADL $^{\#}$ & $16(14-17)$ & $19(15-28)$ & 0.031 \\
SOBDA & $1.1(1.0-1.3)$ & $1.2(1.0-2.0)$ & 0.094
\end{tabular}

Data are presented as $\mathrm{n}(\%)$ or median (interquartile range), unless otherwise stated. ACQ: Asthma Control Questionnaire; CCQ: Clinical COPD (chronic obstructive pulmonary disease) Questionnaire; SGRQ: St George's Respiratory Questionnaire; LCADL: London Chest Activity of Daily Living questionnaire; SOBDA: Shortness of Breath with Daily Activities questionnaire. " : total score.

In this study we explored the occurrence of dynamic hyperinflation in moderate to severe asthma and investigated its relationship with respiratory symptoms and limitations in daily life activities. Although most studies on dynamic hyperinflation are performed in the COPD population, a few studies reported the presence of dynamic hyperinflation in asthma, mostly provoked by methacholine or exercise testing. These studies showed that dynamic hyperinflation was associated with reduced exercise capacity [9, 27] and inconsistent levels of dyspnoea during testing $[8,28,29]$. Interestingly, dynamic hyperinflation might be especially relevant in asthma patients with more severe disease. In a study comparing asthma patients with and without a near-fatal asthma attack, the degree of dynamic hyperinflation during exercise tended to be higher in the patients with near-fatal asthma [30]. It has been shown that severe asthma patients develop dynamic hyperinflation during exercise comparable to COPD patients, a phenomenon that according to the authors might add some insight into the mechanism of daily exercise limitations in this population [10]. Moreover, in a small group of severe asthma patients, dynamic hyperinflation was shown to be treatable as it decreased after omalizumab therapy [13]. Unfortunately, in these studies no data were provided on the relationship of dynamic hyperinflation with respiratory symptoms and limitations of daily life activities.
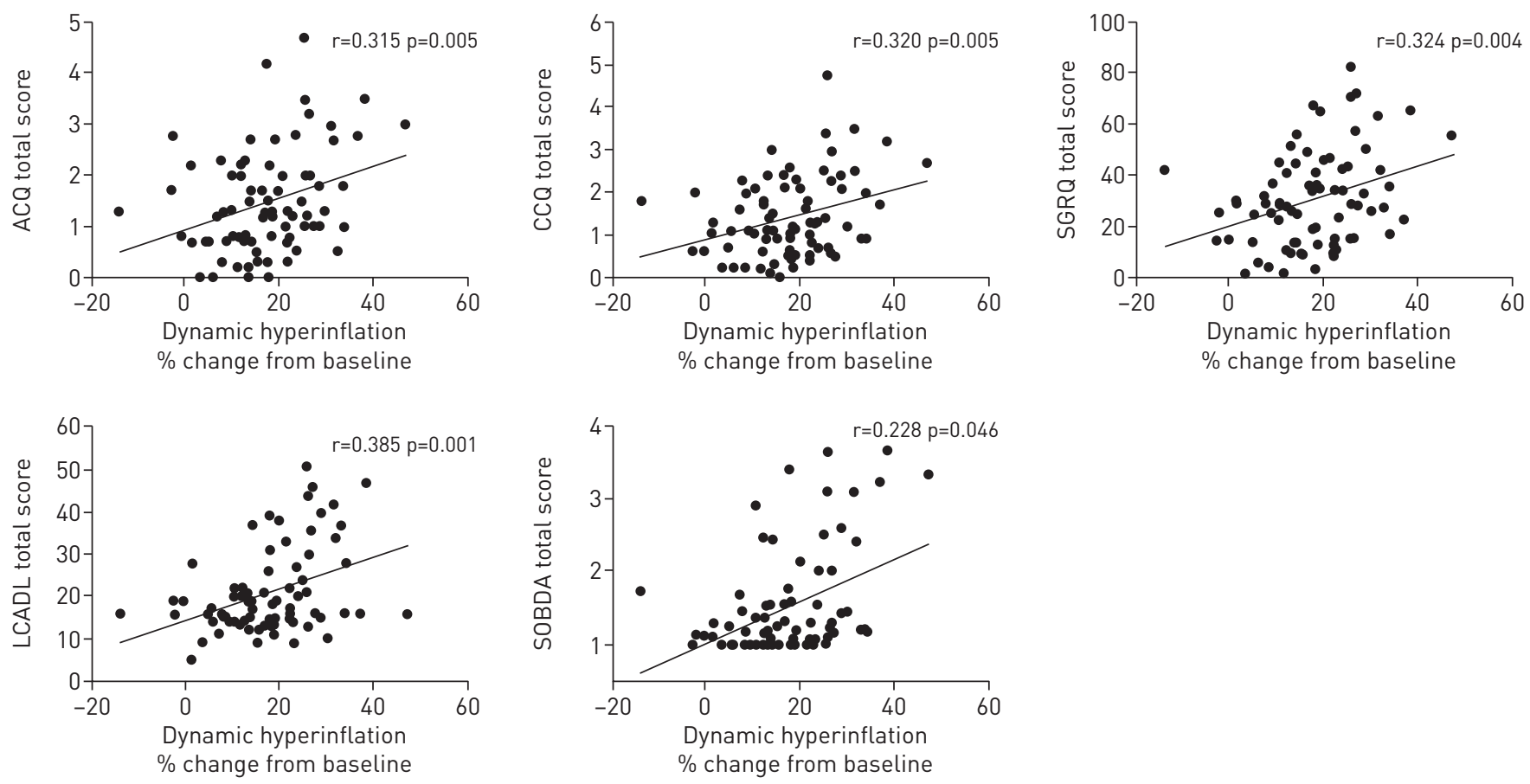

FIGURE 1 Relationship of dynamic hyperinflation and questionnaires. $r$ : correlation coefficient, with corresponding $p$-value. ACQ: Asthma Control Questionnaire; CCQ: Clinical COPD (chronic obstructive pulmonary disease) Questionnaire; SGRQ: St George's Respiratory Questionnaire; LCADL: London Chest Activity of Daily Living questionnaire; SOBDA: Shortness of Breath with Daily Activities questionnaire. 
TABLE 3 The association between degree of dynamic hyperinflation $(\Delta \mathrm{IC})$ and the different questionnaire scores

\begin{tabular}{lcc} 
& $\boldsymbol{\beta}(\Delta \mathrm{CC}) \%(95 \% \mathrm{Cl})$ & p-value \\
\hline CCQ $^{\#}$ & $0.010(-0.004-0.024)$ & 0.155 \\
SGRQ $^{\#}$ & $0.139(-0.135-0.414)$ & 0.315 \\
LCADL $^{\#}$ & $0.198(0.005-0.391)$ & 0.044 \\
SOBDA $^{\#}$ & $0.016(0.004-0.028)$ & 0.009
\end{tabular}

Linear regression analyses adjusted for asthma severity parameters (inhaled corticosteroid dose, percentage of predicted forced expiratory volume in $1 \mathrm{~s}$ and Asthma Control Questionnaire score). $\Delta \mathrm{IC}$ : reduction in inspiratory capacity (=dynamic hyperinflation) measured as the difference between IC at baseline and IC following metronome-paced tachypnoea; CCQ: Clinical COPD Ichronic obstructive pulmonary diseasel Questionnaire; SGRQ: St George's Respiratory Questionnaire; LCADL: London Chest Activity of Daily Living questionnaire; SOBDA: Shortness of Breath with Daily Activities questionnaire. \#. total score.

Our results confirm and extend previous studies by showing that dynamic hyperinflation is highly prevalent in moderate to severe asthma and related to important patient-related outcomes in daily life.

In the current study we found a high proportion of patients showing MPT-induced dynamic hyperinflation, which might be explained by several factors. Firstly, we purposely chose to include only nonobese asthma patients with documented airway obstruction, and without overt smoking-related COPD. However, we cannot exclude that in other subgroups of asthma, e.g. those without airway obstruction or those with obesity, the proportion of patients showing dynamic hyperinflation and the clinical relevance of dynamic hyperinflation might be different. Secondly, we used MPT to measure dynamic hyperinflation. In COPD dynamic hyperinflation is commonly assessed by measuring changes in inspiratory capacity during cardiopulmonary exercise testing (CPET) [31], a complex and laborious test. MPT, a far simpler and less strenuous surrogate of CPET, has been shown in COPD patients to have a good overall accuracy to identify subjects who are susceptible to developing dynamic hyperinflation during CPET and during activities in daily life $[32,33]$. In asthma, there are no studies on the role of dynamic hyperinflation in daily life activities and on the superiority of CPET or MPT to measure it. Our study showed a good coherence between MPT-induced dynamic hyperinflation and asthma symptoms during daily activities. This suggests that a simple MPT measurement might be useful to predict the clinical effects of dynamic hyperinflation in daily practice.

The relationship between limitations in daily life activities and dynamic hyperinflation, irrespective of level of airway obstruction, suggests a role for small-airway dysfunction. In asthma, inflammation and remodelling has been demonstrated in central as well as peripheral airways and there is growing evidence that small airway pathology is more extensive and clinically relevant in patients with severe disease [34-36]. Small-airway abnormalities, whether due to remodelling, transient obstruction or ongoing inflammation contribute to increased airflow limitation and may lead to premature airway closure, air trapping and eventually dynamic hyperinflation [37,38]. Several studies have shown small-airway dysfunction to be associated with poorly controlled asthma and asthma exacerbations [39, 40]. Our results further contribute to the clinical relevance of small-airway dysfunction by showing the relationship between dynamic hyperinflation and limitations in daily life activities.

Interestingly, in severe asthma, the level of exhaled alveolar nitric oxide is closely related to air trapping and airway closure [11], supporting the theory that peripheral airway inflammation and functional abnormalities are interrelated. Ongoing eosinophilic inflammation in the small airways might underlie the demonstrated dynamic hyperinflation in our patients, for which treatment with inhaled corticosteroids might be insufficient. In line with this, a recent pilot study evaluating the usefulness of CPET in verifying and quantifying symptomatic changes following omalizumab treatment showed significant improvements in dynamic hyperinflation as well as exercise capacity [13]. Previous and current results suggest that the identification of small airways disease is not merely speculative, but carries pathophysiological and therapeutic implications [36].

Our results may have important implications for research as well as asthma management in daily practice. The present study provides evidence that dynamic hyperinflation is one of the factors contributing to asthma symptoms and impaired daily life activity. Importantly, FEV1 was not related to questionnaire scores, in line with previous findings that FEV1 cannot be used to predict exertional symptoms in asthma [29]. These findings encourage the development of questionnaires capturing a broader panel of airway 
symptoms and the monitoring of dynamic hyperinflation in asthma management. We showed that a relatively simple measurement of MPT-induced dynamic hyperinflation may be used as an objective parameter significantly linked to patients' subjective reporting of activity limitation. So, MPT testing may provide an additional and useful tool to assess and verify the individual clinical response to treatment, in daily practice as well as in clinical studies. It remains intriguing what mechanisms underlie the development of dynamic hyperinflation in subsets of patients with asthma. Whether the demonstrated dynamic hyperinflation and related asthma symptoms are the consequence of ongoing inflammation in the peripheral airways and might be reversed by systemic anti-inflammatory treatment needs to be investigated.

In conclusion, in the present prospective study we have shown that in moderate to severe asthma the proportion of patients showing dynamic hyperinflation is high. Dynamic hyperinflation is associated with poorer overall health, lower wellbeing and impaired activities of daily life. Therefore, dynamic hyperinflation is an important target for treatment in moderate to severe asthma.

Author contributions: A-N. van der Meer contributed to development of the study design, subject recruitment, collecting study data, performed statistical analysis and wrote the manuscript. K. de Jong contributed to statistical analysis, interpretations and manuscript preparation. A. Hoekstra-Kuik performed the pulmonary function tests and MPT measurement and contributed to the manuscript preparation. E.H. Bel and A. ten Brinke contributed to the development of the study design, statistical analysis and manuscript preparation.

Conflict of interest: A-N. van der Meer reports grants from Medical Centre Leeuwarden Research Fund, TEVA, GSK and Stichting Longgeneeskunde Fryslan, during the conduct of the study. K. de Jong has nothing to disclose. A. Hoekstra-Kuik has nothing to disclose. E.H. Bel reports grants and/or personal fees from Novartis, GSK, Sanofi Regenron, Roche, AstraZeneca, Teva, Veactira and Boehringer, outside the submitted work. A. ten Brinke reports grants from Medical Centre Leeuwarden Research Fund, TEVA, GSK and Stichting Longgeneeskunde Fryslan, during the conduct of the study; institutional fees for research advisory board work from GSK, Sanofi, TEVA, AstraZeneca and Boehringer Ingelheim, and institutional fees for lecturing from GSK, TEVA and AstraZeneca, outside the submitted work.

Support statement: This study was supported by unrestricted grants from Medical Centre Leeuwarden research fund, Stichting Longgeneeskunde Fryslân, GlaxoSmithKline and Teva. Funding information for this article has been deposited with the Crossref Funder Registry.

\section{References}

1 Wenzel SE. Asthma phenotypes: the evolution from clinical to molecular approaches. Nat Med 2012; 18: 716-725.

2 Foster JM, McDonald VM, Guo M, et al. "I have lost in every facet of my life": the hidden burden of severe asthma. Eur Respir J 2017; 50: 1700765.

3 Weatherald J, Lougheed MD, Taillé C, et al. Mechanisms, measurement and management of exertional dyspnoea in asthma. Eur Respir Rev 2017; 26: 170015.

4 Guenette JA, Webb KA, O’Donnell DE. Does dynamic hyperinflation contribute to dyspnoea during exercise in patients with COPD? Eur Respir J 2012; 40: 322-329.

5 O'Donnell DE, Travers J, Webb KA, et al. Reliability of ventilatory parameters during cycle ergometry in multicentre trials in COPD. Eur Respir J 2009; 34: 866-874.

6 Garcia-Rio F, Lores V, Mediano O, et al. Daily physical activity in patients with chronic obstructive pulmonary disease is mainly associated with dynamic hyperinflation. Am J Respir Crit Care Med 2009; 180: 506-512.

7 Laveneziana P, Lotti P, Coli C, et al. Mechanisms of dyspnoea and its language in patients with asthma. Eur Respir J 2006; 27: 742-747.

8 Lougheed MD, Fisher T, O'Donnell DE. Dynamic hyperinflation during bronchoconstriction in asthma: implications for symptom perception. Chest 2006; 130: 1072-1081.

9 Kosmas EN, Milic-Emili J, Polychronaki A, et al. Exercise-induced flow limitation, dynamic hyperinflation and exercise capacity in patients with bronchial asthma. Eur Respir J 2004; 24: 378-384.

10 Benfante A, Di Marco F, Terraneo S, et al. Dynamic hyperinflation during the 6-min walk test in severely asthmatic subjects. ERJ Open Res 2018; 4: 00143-2017.

11 van Veen IH, Sterk PJ, Schot R, et al. Alveolar nitric oxide versus measures of peripheral airway dysfunction in severe asthma. Eur Respir J 2006; 27: 951-956.

12 de Groot JC, Storm H, Amelink M, et al. Clinical profile of patients with adult-onset eosinophilic asthma. ERJ Open Res 2016; 2: 00100-2015.

13 Schäper C, Gläser S, Felix SB, et al. Omalizumab treatment and exercise capacity in severe asthmatics - results from a pilot study. Respir Med 2011; 105: 3-7.

14 Ferreira PG, Freitas PD, Silva AG, et al. Dynamic hyperinflation and exercise limitations in obese asthmatic women. J Appl Physiol 2017; 123: 585-593.

15 Ortega HG, Liu MC, Pavord ID, et al. Mepolizumab treatment in patients with severe eosinophilic asthma. $N$ Engl J Med 2014; 371: 1198-1207.

16 Chupp GL, Bradford ES, Albers FC, et al. Efficacy of mepolizumab add-on therapy on health-related quality of life and markers of asthma control in severe eosinophilic asthma (MUSCA): a randomised, double-blind, placebo-controlled, parallel-group, multicentre, phase 3b trial. Lancet Respir Med 2017; 5: 390-400.

17 Global Initiative for Asthma (GINA). Global Strategy for Asthma Management and Prevention. 2015. Available from: http://ginasthma.org/ 
18 Juniper EF, O'Byrne PM, Guyatt GH, et al. Development and validation of a questionnaire to measure asthma control. Eur Respir J 1999; 14: 902-907.

19 van der Molen T, Willemse BWM, Schokker S, et al. Development, validity and responsiveness of the Clinical COPD Questionnaire. Health Qual Life Outcomes 2003; 1: 13.

20 Jones PW, Quirk FH, Baveystock CM, et al. A self-complete measure of health status for chronic airflow limitation. The St. George's Respiratory Questionnaire. Am Rev Respir Dis 1992; 145: 1321-1327.

21 Garrod R, Bestall JC, Paul EA, et al. Development and validation of a standardized measure of activity of daily living in patients with severe COPD: The London chest activity of daily living scale (LCADL). Respir Med 2000; 94: 589-596.

22 Watkins ML, Wilcox TK, Tabberer M, et al. Shortness of Breath with Daily Activities questionnaire: validation and responder thresholds in patients with chronic obstructive pulmonary disease. BMJ Open 2013; 3: e003048.

23 American Thoracic Society, European Respiratory Society. ATS/ERS recommendations for standardized procedures for the online and offline measurement of exhaled lower respiratory nitric oxide and nasal nitric oxide, 2005. Am J Respir Crit Care Med 2005; 171: 912-930.

24 Miller MR, Hankinson J, Brusasco V, et al. Standardisation of spirometry. Eur Respir J 2005; 26: 319-338.

25 Gelb AF, Gutierrez CA, Weisman IM, et al. Simplified detection of dynamic hyperinflation. Chest 2004; 126 $1855-1860$.

26 Lahaije AJMC, Willems LM, van Hees HW, et al. Diagnostic accuracy of metronome-paced tachypnea to detect dynamic hyperinflation. Clin Physiol Funct Imaging 2013; 33: 62-69.

27 Murariu C, Ghezzo H, Milic-Emili J, et al. Exercise limitation in obstructive lung disease. Chest 1998; 114: 965-968.

28 van der Wiel E, Postma DS, van der Molen T, et al. Effects of small airway dysfunction on the clinical expression of asthma: a focus on asthma symptoms and bronchial hyper-responsiveness. Allergy 2014; 69: 1681-1688.

29 Vermeulen F, Garcia G, Ninane V, et al. Activity limitation and exertional dyspnea in adult asthmatic patients: what do we know? Respir Med 2016; 117: 122-130.

30 Barreiro E, Gea J, Sanjuás C, et al. Dyspnoea at rest and at the end of different exercises in patients with near-fatal asthma. Eur Respir J 2004; 24: 219-225.

31 O'Donnell DE, Revill SM, Webb KA. Dynamic hyperinflation and exercise intolerance in chronic obstructive pulmonary disease. Am J Respir Crit Care Med 2001; 164: 770-777.

32 Lahaije A, van Helvoort H, Dekhuijzen R, et al. Can COPD patients who hyperinflate during daily life activities be identified by laboratory tests? Respiration 2013; 86: 237-242.

33 Lahaije AJMC, van Helvoort HAC, Dekhuijzen PNR, et al. Resting and ADL-induced dynamic hyperinflation explain physical inactivity in COPD better than FEV1. Respir Med 2013; 107: 834-840.

34 Balzar S, Wenzel SE, Chu HW. Transbronchial biopsy as a tool to evaluate small airways in asthma. Eur Respir J 2002; 20: 254-259.

35 Berry M, Hargadon B, Morgan A, et al. Alveolar nitric oxide in adults with asthma: evidence of distal lung inflammation in refractory asthma. Eur Respir J 2005; 25: 986-991.

36 Contoli M, Santus P, Papi A. Small airway disease in asthma: pathophysiological and diagnostic considerations. Curr Opin Pulm Med 2015; 21: 68-73.

37 Wagner EM, Liu MC, Weinmann GG, et al. Peripheral lung resistance in normal and asthmatic subjects. Am Rev Respir Dis 1990; 141: 584-588.

38 Ueda $\mathrm{T}$, Niimi A, Matsumoto $\mathrm{H}$, et al. Role of small airways in asthma: investigation using high-resolution computed tomography. J Allergy Clin Immunol 2006; 118: 1019-1025.

39 Bourdin A, Paganin F, Préfaut C, et al. Nitrogen washout slope in poorly controlled asthma. Allergy 2006; 61: 85-89.

40 Pisi R, Tzani P, Aiello M, et al. Small airway dysfunction by impulse oscillometry in asthmatic patients with normal forced expiratory volume in the 1st second values. Allergy Asthma Proc 2013; 34: e14-e20. 